\title{
Preliminary Results Towards Fidelity Evaluation of an In-flight Simulator
}

\author{
Benjamin Fragnière ${ }^{1}$, Johannes Wartmann ${ }^{1}$ and Steffen Greiser ${ }^{1}$ \\ DLR (German Aerospace Center), \\ Institute of Flight Systems \\ Lilienthalplatz 7, 38108 Braunschweig, Germany
}

This paper presents the analysis of the fidelity obtained when performing in-flight simulations of the Bo105 helicopter in forward flight with the Active Control Technology/ Flying Helicopter Simulator (ACT/FHS). The results are preliminary in the sense that they are based on the real-time and fully non-linear simulation model of the ACT/FHS in DLR's helicopter simulator and not in the ACT/FHS itself. Yet, a flight test proven controller setting is used in the simulations. The ACT/FHS, the model-based controller, and the command model are briefly presented. The precision of the MBC for each control/response axis is evaluated quantitatively through frequency-domain methods, time-domain methods and handling quality analysis. A perceived fidelity evaluation process based on piloted simulations and comparative flight tests with the Bo105 is presented. This process is first applied to evaluate the model-following precision and second to evaluate the fidelity of the in-flight simulation. The results of the evaluations are used to identify strengths and weaknesses of the in-flight simulation for each response axis, including cross-couplings.

\section{Nomenclature}

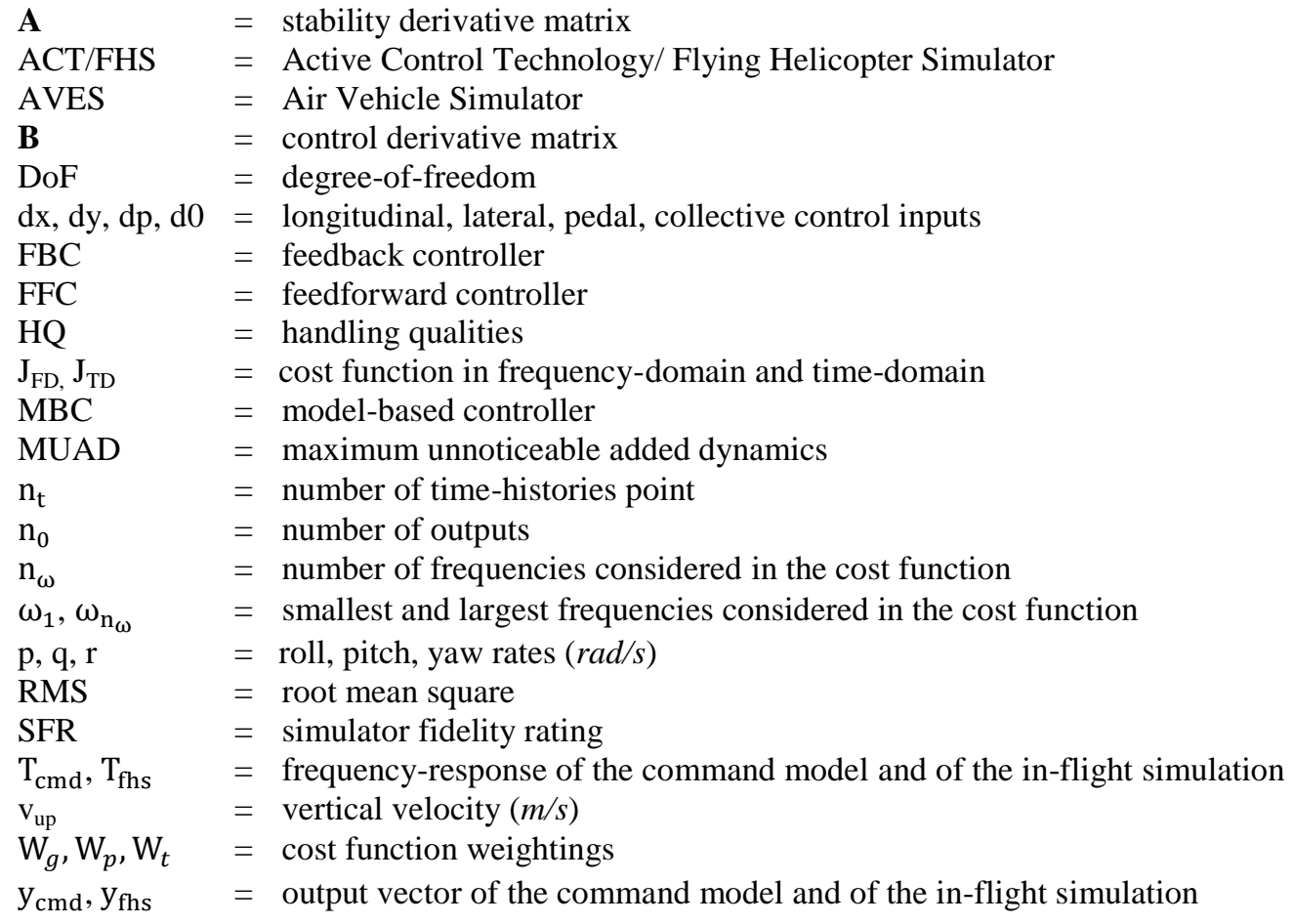

\section{Introduction}

When speaking of in-flight simulation, it is important to make the distinction between the terms in-flight simulator and variable-stability helicopter. A variable-stability helicopter is a generic term describing a helicopter having the capability to vary in flight its handling qualities (HQ) characteristics. When considering only slow dynamics, such capability can be reached using a low bandwidth controller, such as an autopilot. This was for

\footnotetext{
${ }^{1}$ Research Scientist, Dept. FT-HUB.
} 
example the case for the first variable-stability helicopter, the NASA Langley HO3S-1, in the 1950's. The variable stability was achieved for the pitch, roll and yaw axes using a modified autopilot and tunable potentiometers [1]. An in-flight simulator goes further in the sense that it requires the capability to follow a given response-type in all axes and to frequencies up to typically $10 \mathrm{rad} / \mathrm{s}$ or more. To achieve this, modern in-flight simulators, such as the RASCAL (a modified UH-60A) of the US Army [2], the ASRA (a modified Bell-414) of the NRC [3] or the ACT/FHS (a modified EC-135) of the DLR [4], are equipped with full authority, 4-axes controlled, fly-by-wire/flyby-light control systems and extensive sensor systems. The flight control system also requires a precise feed-forward controller since feedback controllers alone usually cannot reach the desired bandwidth of $10 \mathrm{rad} / \mathrm{s}$ or more. Therefore, an accurate (inverse) model of the in-flight simulator is necessary, including all the cross-coupling responses at high frequency. In-flight simulators can be used in a broad range of topics such as HQ research, flight controls research, system development or flight crew training.

The focus of the present paper is placed on the fidelity evaluation of the in-flight simulation. While the fidelity level of ground simulators has been widely investigated, it is not the case for in-flight simulators. Most of the literature related to in-flight simulation shows results of studies made using in-flight simulators and not how good desired flight dynamics can be actually reproduced by the in-flight simulator. The few exceptions found in the literature include the simulation of the Lynx helicopter at 60 knots using the ATTHeS in-flight simulator [5,6] and the simulation of generic decoupled transfer functions using the RASCAL helicopter [7]. This paper goes beyond the latter by simulating a fully coupled real helicopter and beyond the former by providing a more detailed analysis of the in-flight simulator fidelity (predicted and perceived fidelity evaluation, on- and off-axis responses, timedomain, frequency-domain and HQ criteria). The paper is structured as follows: in Section III, the in-flight simulator ACT/FHS with its model-based controller (MBC) is briefly presented; the command model used in the MBC, a 6degrees-of-freedom (DoF) of the Bo-105 helicopter in forward flight, is described in Section IV; the methods and results of the in-flight simulation evaluation are presented and discussed in Section V.

\section{ACT/FHS In-Flight Simulator}

\section{A. Active Controller Simulator/ Flying Helicopter Simulator (ACT/FHS)}

The research helicopter ACT/FHS (Active Control Technology/Flying Helicopter Simulator) is in service at DLR since 2002. It is a highly modified EC135 manufactured by Airbus Helicopters (formerly Eurocopter). In addition to the standard instrumentation, the ACT/FHS is equipped with additional sensors such as noseboom, Honeywell IMU and GPS. Most of the sensors are redundant, meaning that sensor failures are compensated by others. All signals such as control inputs and measured flight dynamical states are stored to allow evaluations afterwards; more details are given in [4]. One major modification is related to the control system. The original mechanical controls were extended with a full authority fly-by-wire/fly-by-light control system. In all piloting modes (i.e. safety pilot mode, direct and experimental mode), the electrical flight control system is active and the mechanical control system serves as backup system. Full authority control system means, that the control inputs' are transmitted with high rates (up to almost 200\%/s) for the full range of travel between $0 \%$ and $100 \%$. These fast and large swashplate control deflections are realized with smart hydraulic actuators which are specific for the ACT/FHS. In addition, time delays between commanded control inputs and swashplate deflections are small (approx. $50 \mathrm{ms)}$ compared to typical target control bandwidths (usually below $10 \mathrm{rad} / \mathrm{s}$ for the ACT/FHS). Experimental flight control systems may take advantage of this feature as a high-bandwidth might be achieved.

In-flight simulation imposes a specific dynamical behavior to the ACT/FHS. This might require a highbandwidth control system so that the ACT/FHS dynamics are widely changed. Hence, a high-fidelity in-flight simulation benefits from high rates and large control deflections. This control system was developed in several DLR projects such as PAVE [8], ALLFlight [9], and ALL-in-Flight [10]. In each of these projects, the ACT/FHS was a vital component for development and flight tests. The overall result is a model-based controller (MBC) which is presented in more detail thereafter. The MBC was first developed and tested successfully in PAVE for short time periods. During ALLFlight the model-based control featured maneuvering capability for longer time periods which was possible by re-designing the feedforward and feedback control system. ALL-in-Flight then achieves a highbandwidth control system especially in pitch and roll axes by extending the closed-loop controller with acceleration feedback. These developments serve as basis for the research on in-flight simulation. The next section will thus present an overview about the model-based control.

\section{B. Model-based controller}

The model-based controller (MBC) is part of the experimental system of the ACT/FHS. The evaluation pilot does not control the ACT/FHS actuators directly, but virtually by piloting the command model. The MBC calculates 
the control signals sent to the actuators so that the ACT/FHS follows the commanded model states. The structure of the MBC is shown in Fig. 1 and the different components are described in the following.

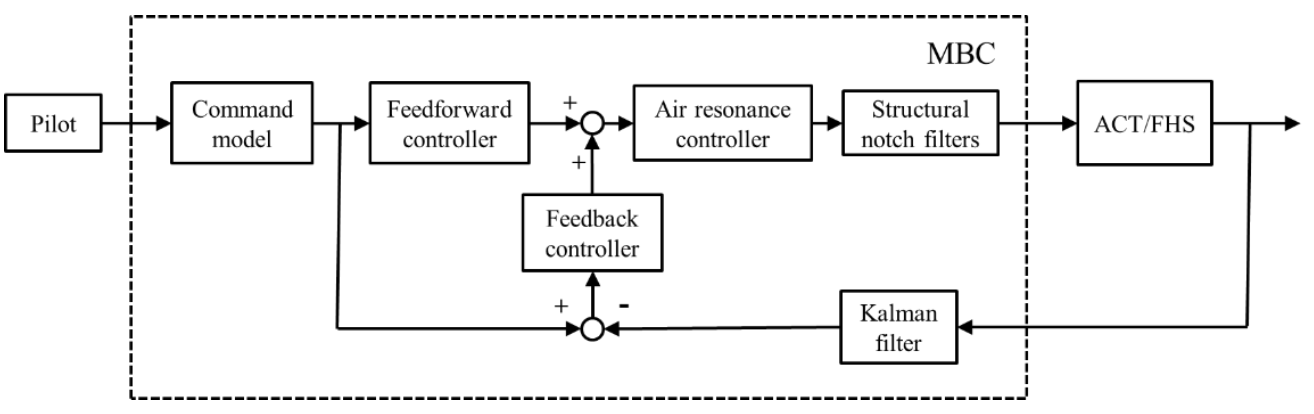

Fig. 1 Structure of the model-based controller (MBC).

The command model provides the reference flight dynamics that have to be followed by the ACT/FHS. It consists in the online simulation of a given model piloted by the evaluation pilot. The output of the simulation is fed into the feedforward and feedback controllers that command the actuators of the ACT/FHS. The command model is described in detail in the next section.

The feedforward controller (FFC) calculates the appropriate actuator positions that allow the ACT/FHS to follow the reference flight dynamics of the command model. It consists of the inverse of a state-space model of the ACT/FHS flight dynamics. As four control axes are available, only four states can be perfectly followed by the feedforward controller; here the vertical velocity, yaw rate, roll rate, and pitch rate are followed. The ACT/FHS model used in the FFC is a 9-DoF model accounting for rigid body dynamics, (implicit) rotor flapping, and dynamic inflow. Five ACT/FHS models are used for five trim velocities ranging from hover to 120 knots. A weighted superposition of the respective outputs is performed based on the airspeed. A high pass filter ensures that the output of the FFC decreases to zero at low frequencies. The methods used to calculate the inverse dynamics of the ACT/FHS model are described in detail in [11] and [12].

The feedback controller (FBC) provides additional actuator commands accounting for the errors between the reference states of the command model and the actual states of the ACT/FHS. The errors arise from imperfections of the inverse dynamics in the FFC and from external disturbances. The FBC consists in four separated PI controllers for the pitch, roll, yaw and heave axes. An anti-windup scheme is integrated with each integrator. In addition to the rotational rates and attitudes, the vertical, forward and lateral velocities, the altitude, track and slip angle can be controlled. For this study, the altitude controller is turned off.

An air resonance controller is implemented under the form of an additional control input by considering the regressive lead-lag as a disturbance [13]. The inputs of the air resonance controller are the roll rate and acceleration; the output is an additional lateral control input that suppresses the $1.8 \mathrm{~Hz}$ roll oscillation. Structural notch filters allow limiting the excitation of the structural modes of the ACT/FHS. The filtered frequencies depend on the axis but are all in the frequency range of 20-70 rad/s. All measurements are processed with a Kalman-Filter that on the one hand reconstructs missing information and on the other hand fuses redundant sensor information to one single output. These filtered measurements provide the MBC with all required states.

\section{Command Model}

The command model describes the desired behavior of the helicopter. It is piloted by the evaluation pilot and provides the reference states to the feedforward and feedback controllers. In the case of a normal operating helicopter, the command model is typically designed according to certain HQ criteria, such as ADS-33 [14]. In the case of an in-flight simulation, the command model is a flight dynamics model of the helicopter to be simulated.

\section{A. Model structure}

The command model chosen in this study is a quasi-nonlinear, 6-DoF helicopter model accounting for rigid body dynamics only. Fig. 2 gives an overview of the structure of the command model. The Euler terms, the gravity forces and the trim curves are implemented in their nonlinear form. The aerodynamic forces are reduced to a linearized representation around a trim state. The linear part of the model is given by the stability derivative matrix $\mathbf{A}$ and the control derivative matrix B. An equivalent time delay for each input axis accounts for the neglected high order dynamics. Any 6-DoF flight dynamics model can be implemented in this structure by simply changing the $\mathbf{A}$ and $\mathbf{B}$ matrices and the equivalent time delay. The model is only valid in the vicinity of the trim state corresponding to the 
$\mathbf{A}$ and $\mathbf{B}$ matrices. The trim states used in the command model are not the ones of the simulated helicopter but the ones of the ACT/FHS.

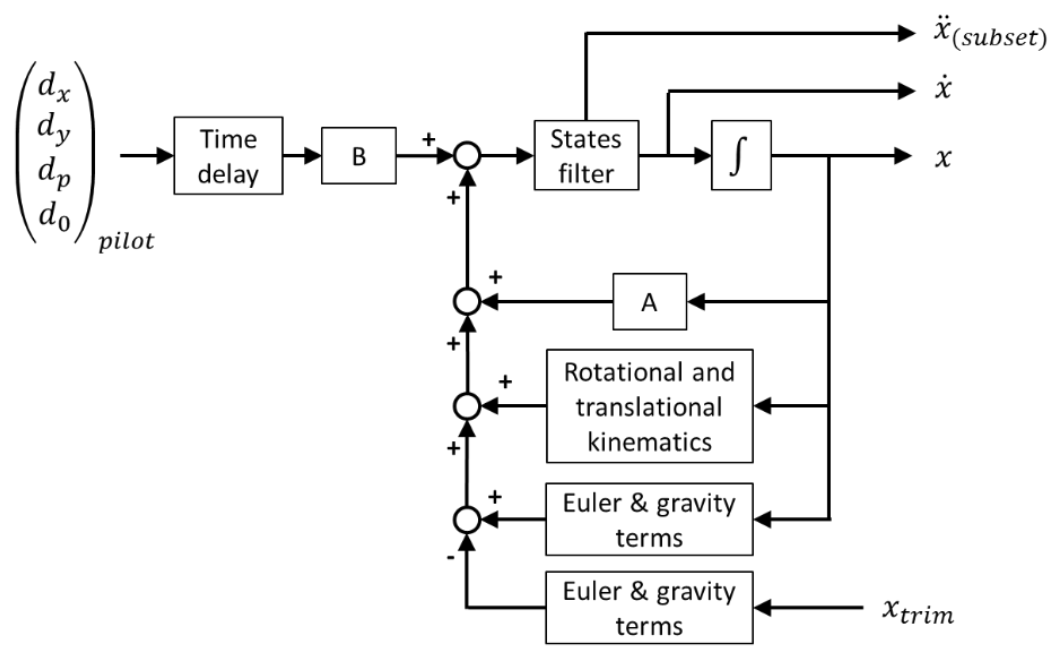

Fig. 2. Structure of the command model.

The feedforward controller is the dynamic inverse of the identified ACT/FHS state-space model including the longitudinal flapping, lateral flapping and dynamic inflow modes, while the command model only accounts for the rigid body dynamics. Trying to exactly follow the rigid body dynamics of the command model would generate exaggeratedly high amplitude actuator peaks in the ACT/FHS to compensate for the high frequency modes included in the feedforward model. As high amplitude actuator peaks should be avoided, the states are filtered in the command model.

\section{B. Model data and characteristics}

The choice of the Bo-105 for the command model is due to the availability of this helicopter type at DLR; flight tests can be performed for evaluation purposes. The model chosen for this study is the 6-DoF quasi-nonlinear model of the Bo-105 at 80 knots published in 1989 in [15]. It is the result of a system identification process performed by the Aeroflightdynamics Directorate of the US Army. The identification was done based on flight data coming from the DLR Bo-105 research helicopter and using frequency-domain methods. Some of the main physical characteristics of the Bo-105 are given in Table 1 in comparison to the ACT/FHS. The poles of the Bo-105 model at 80 knots and ACT/FHS model at 90 knots are shown in Fig. 3. It can be seen that the fast poles (pitch and roll) of the Bo-105 are faster and more coupled than the ones of the ACT/FHS.

\begin{tabular}{lll} 
& Bo-105 & ACT/FHS \\
\hline Maximum take-off weight & $2300 \mathrm{~kg}$ & $2835 \mathrm{~kg}$ \\
Main rotor radius & $4.91 \mathrm{~m}$ & $5.10 \mathrm{~m}$ \\
Equivalent hinge offset & $14 \%$ & $8.7 \%$ \\
\hline
\end{tabular}

Table 1 Physical characteristics of the simulated helicopter (Bo-105) and the in-flight simulator (ACT/FHS).
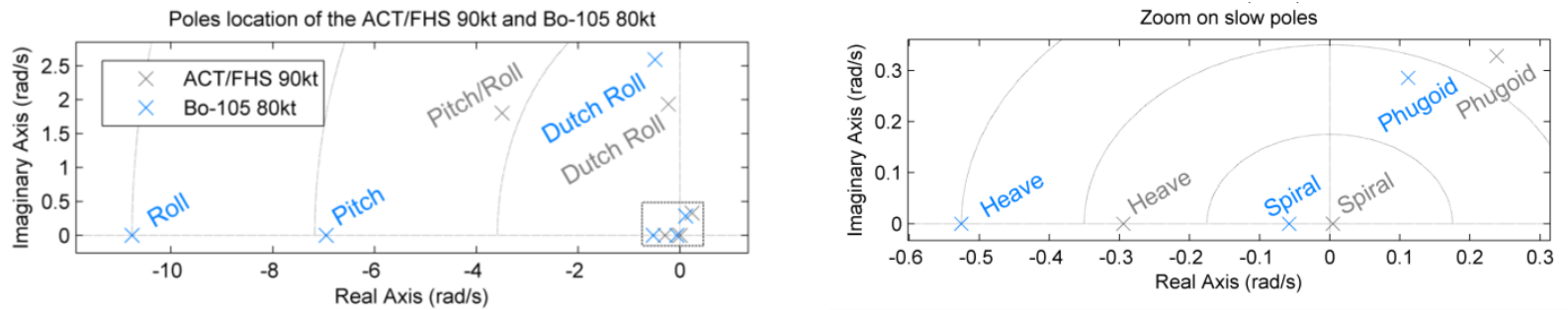

Fig. 3 Poles location of the Bo-105 at 80 knots and ACT/FHS at 90 knots. 


\section{Command model tuning}

Before testing the in-flight simulation capability, the command model was tested and tuned during piloted simulations. An evaluation based on flight test data was not possible, due to the two following reasons: first the data of the identification campaign performed in the 1980' were not available; second the Bo-105 helicopter currently used at DLR is not equipped with measurement and recording system. For the piloted simulations the Bo-105 command model has been implemented as standalone in the AVES [16] simulator. A helicopter pilot with extensive and recent Bo-105 flight experience was asked to perform standard flight maneuvers and to report the clear dissimilarities observed against to the real Bo-105 helicopter. In particular, the following characteristics have been observed: short term and long term on-axis response time, amplitude and direction of the off-axis responses (crosscoupling) and control sticks' displacements. These characteristics have been chosen because they can be easily noticed by the pilot and associated to control and stability derivatives. The initial model dynamics showed three main weaknesses concentrated on the off-axes: (1) the heave response during acceleration/deceleration maneuvers, (2) the couplings from the heave/collective to pitch/roll/yaw and (3) the coupling from yaw to pitch were not realistic. Additionally, corrective factors had to be added on the longitudinal cyclic and collective sticks to account for the geometrical difference between the sticks of the Bo-105 and the sticks of the ACT/FHS. After several iterations of model evaluation and parameter tuning, the pilot considered the Bo- 105 model as realistic, in the sense that he was not able to tell in which direction the characteristics shall be changed.

Interestingly, while the derivatives in the command model were taken originally from the frequency-domain identification in [15], most of the derivative modified during the tuning process were much closer to the timedomain identification results in [15] at the end.

\section{In-Flight Simulation Fidelity Evaluation}

This section describes how the fidelity of the in-flight simulation has been evaluated. The evaluation was limited to flight dynamics and not extended to the whole simulator fidelity. Fidelity of the visual cueing, motion cueing, audio cueing, cockpit arrangement and controls loading were not investigated. Fig. 4 shows the four cases considered for the evaluations:

Test case A is the reference case: the Bo- 105 .

Test case B is the direct simulation of the 6-DoF Bo-105 model seen in the previous section,

Test case $C$ is the in-flight simulation simulated in the AVES [16] simulator, i.e. the simulation of the complete MBC applied to the fully non-linear model of the ACT/FHS,

Test case D is the normal in-flight simulation, i.e. the ACT/FHS in flight controlled by the MBC; this case has not yet been tested and is shown for completeness.

Each case has its own flight dynamics: case B differs from case A by the modeling error of the 6-DoF model of the Bo-105. Case C differs from case B by the model-following error of the MBC. Case D differs from case C by the modeling error of the fully non-linear model of the ACT/FHS.

The evaluation process described in this paper consists in the two following steps:

1) The evaluation of the model-following precision, by comparing the flight dynamics in cases $\mathrm{B}$ and $\mathrm{C}$ of Fig. 4.

2) The evaluation of the fidelity of the in-flight simulation simulated in AVES, by comparing the flight dynamics in cases A and C of Fig. 4.

The final step would consist in evaluating the fidelity of the normal in-flight simulation by comparing the flight dynamics in cases A and D of Fig. 4. This step could not be performed because the ACT/FHS was not available in experimentally controlled mode during the whole time of the study. In this paper, the term in-flight simulation will refer to the in-flight simulation simulated in the AVES simulator, i.e. the case C of Fig. 4.

It is classical to distinguish between predicted fidelity and perceived fidelity [18]. The predicted fidelity is based on data recorded during simulations or during flight tests; it indicates the level of similarity of a selected set of flight dynamics characteristics between the simulation and the simulated helicopter in flight. The perceived fidelity is based on the subjective opinions of the pilots, evaluated during piloted simulation or flight tests through questionnaires. The questionnaires address the level of fidelity of the simulation perceived by the pilots. While both, predicted and perceived fidelity metrics, were used for the first step, only perceived fidelity metrics were applied for the second step due to the absence of measurement and recording system in the Bo-105 helicopter used at the DLR. Each of the simulations performed in the AVES simulator were done on the fixed platform. 

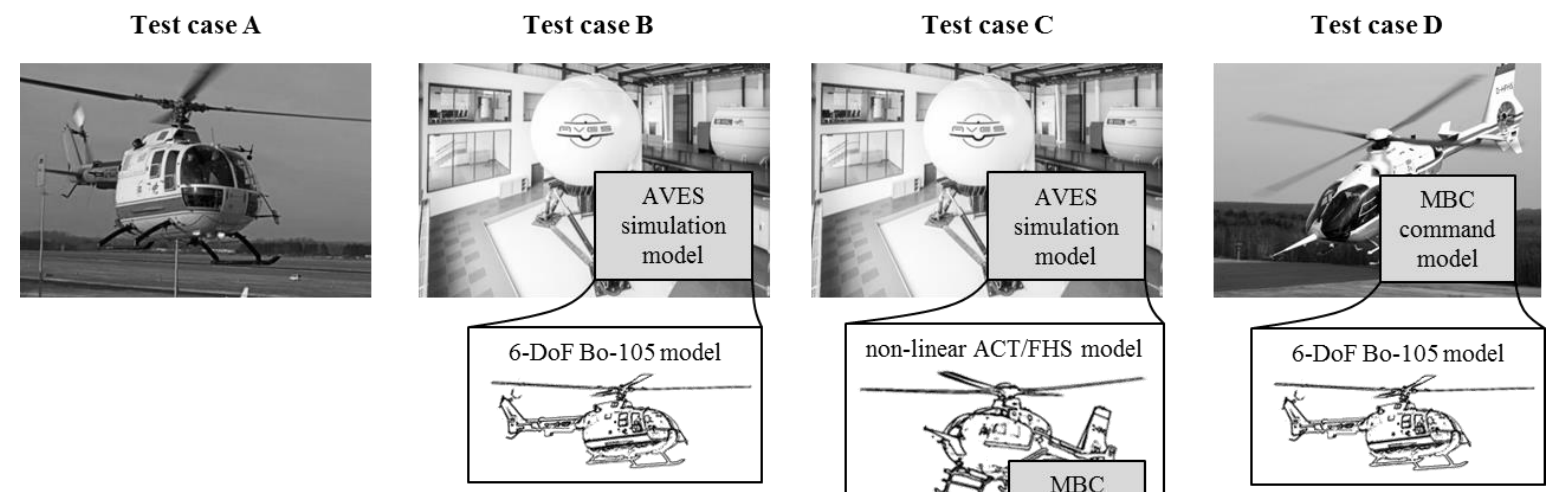

Fig. 4 Overview of the different testing configurations: case $A$ is the Bo-105, the reference case, case $B$ is the direct simulation of the Bo-105 in AVES, case $C$ is the in-flight simulation simulated in AVES, case D is the normal in-flight simulation in the ACT/FHS (this case has not been tested and is shown for completeness).

\section{A. Model-following precision evaluation}

Model-following precision evaluation is an intermediate step for the evaluation of the in-flight simulation fidelity that allows decoupling the quality of the MBC from the quality of the command model. It is obtained by comparing results from the test cases $\mathrm{B}$ and $\mathrm{C}$ shown in Fig. 4. The evaluation has been divided in 16 control/response axes, i.e. all on-axis and off-axis (cross-coupling) responses from the longitudinal cyclic, lateral cyclic, pedal and collective stick inputs to the pitch rate, roll rate, yaw rate and vertical velocity responses. The predictive part of the evaluation relies on analyses in frequency-domain, in time- domain, and in terms of HQ ratings. Therein, the data were simulated by applying computer-generated excitations in the simulation. The modelfollowing precision has also been evaluated subjectively using piloted simulations and perceived fidelity evaluation methods. The evaluation criteria, the respective results, and their discussions are described in the following.

It is important to mention that the feedback controller setting used in this study was not designed for an optimized in-flight simulation performance in the simulator, but it is a flight test proven setting that was used in former flight test campaigns of the ACT/FHS. Hence, those results do not reflect the best achievable performance of the in-flight simulator but rather a benchmark with a realistic controller setting that can be used for future real inflight simulations with the ACT/FHS.

\section{Frequency-domain analysis}

The frequency-responses of the command model and the in-flight simulation have been generated using sweep excitation signals. The Bode diagrams of the mismatch between these two frequency-responses can be seen together with the maximum unnoticeable added dynamics (MUAD) bounds for each control/response axis in Fig. 9 in the Appendix. The MUAD bounds are defined in [19]; they define an acceptable level of mismatch in equivalent systems in term of magnitude and phase difference. It is claimed that two different systems with magnitude and phase differences within a given envelope cannot be distinguished by a pilot. These bounds were initially designed for the pitch control of a fixed-wing aircraft, but were also used for helicopter simulation fidelity evaluation ([20, 21]). The accuracy of these bounds has been questioned in some research [22], but they give at least an approximate and qualitative estimation of the model-following precision.

The model-following cost function as defined in [23] has also been calculated for each control/response axis. This cost function is a weighted average of the squared magnitude error and squared phase error between the commanded frequency-response $\mathrm{T}_{\mathrm{cmd}}$ and the simulated frequency-response $\mathrm{T}_{\mathrm{fhs}}$ :

$$
\mathrm{J}_{\mathrm{FD}}=\frac{20}{\mathrm{n}_{\omega}} \sum_{\omega_{1}}^{\omega_{\mathrm{n}_{\omega}}}\left\{\mathrm{W}_{\mathrm{g}}\left(\left|\mathrm{T}_{\mathrm{fhs}}\right|-\left|\mathrm{T}_{\mathrm{cmd}}\right|\right)^{2}+\mathrm{W}_{\mathrm{p}}\left(\angle \mathrm{T}_{\text {fhs }}-\angle \mathrm{T}_{\mathrm{cmd}}\right)^{2}\right\}
$$


with $|\mathrm{T}|$ the frequency response magnitude, given in $\mathrm{dB}$ and $\angle \mathrm{T}$ the frequency response phase, given in degrees. Standard values for the weights are $\mathrm{W}_{\mathrm{g}}=1$ and $\mathrm{W}_{\mathrm{p}}=0.01745 . \omega_{1}$ and $\omega_{\mathrm{n}_{\omega}}$ are typically set to $1 \mathrm{rad} / \mathrm{s} \mathrm{and} 10 \mathrm{rad} / \mathrm{s}$ for flight-dynamics modelling purposes. According to [23], a cost function $\mathrm{J}_{\mathrm{FD}}<100$ is generally considered as reflecting an acceptable level of accuracy. Some of the individual cost functions can reach a value of $\mathrm{J}_{\mathrm{FD}}<150-$ 200 without resulting in a noticeable loss of overall accuracy. The model-following cost of each control/response axis can be seen in Fig. 5(a); the dark and light green sectors correspond to the upper and lower bound of the aforementioned guideline.

\section{Handling quality analysis}

The comparison of the HQ ratings of the command model and of the in-flight simulation provides another indication of the model-following precision. A set of standard HQ criteria are defined in ADS-33 [14]. Each of the criteria consists in a cross-plot with three sectors indicating a HQ Level 1, 2, and 3. In this study, the following criteria are considered: Attitude quickness, bandwidth, height response, pitch/roll couplings, yaw-collective couplings. The resulting HQ evaluation can be seen in Fig. 8 in the Appendix. From the literature, no guideline exists regarding the level of acceptable HQ mismatch.

\section{Time-domain analysis}

The time-domain analysis is based on the response errors between the command model output and of the inflight simulation results when the models are excited by doublets and 3-2-1-1multistep input signals. The Root Mean Square (RMS) error in time-domain, as defined in [23], is the root mean square of the average weighted error between the commanded output and the simulated output:

$$
\mathrm{J}_{\mathrm{TD}}=\sqrt{\left(\frac{1}{\mathrm{n}_{\mathrm{t}} \cdot \mathrm{n}_{0}}\right) \sum_{\mathrm{i}=1}^{\mathrm{n}_{\mathrm{t}}}\left\{\left(y_{\mathrm{fhs}}-y_{\mathrm{cmd}}\right)^{T} \mathrm{~W}_{t}\left(y_{\mathrm{fhs}}-y_{\mathrm{cmd}}\right)\right\}}
$$

with $\mathrm{n}_{\mathrm{t}}$ the number of time-history points, $\mathrm{n}_{0}$ the number of outputs, $W_{t}$ a weighting matrix. $\mathrm{y}_{\mathrm{cmd}}$ and $\mathrm{y}_{\mathrm{fhs}}$ are the vectors of the commanded states and simulated outputs respectively. The weighting matrix is typically chosen so that the units of the output are converted to $\mathrm{deg}, \mathrm{deg} / \mathrm{s}, \mathrm{ft} / \mathrm{s}$ and $\mathrm{ft} / \mathrm{s}^{2}$. A guideline given in [23] states that a value in the range of $\mathrm{J}_{\mathrm{TD}}<1$ to 2 reflects an acceptable level of accuracy for flight-dynamics modelling.

The RMS error for a sequence of doublets and 3-2-1-1 excitations with varied amplitudes are presented for each control/response axis in Fig. 5(b); the dark and light green sectors correspond to the upper and lower bound of the aforementioned guideline.

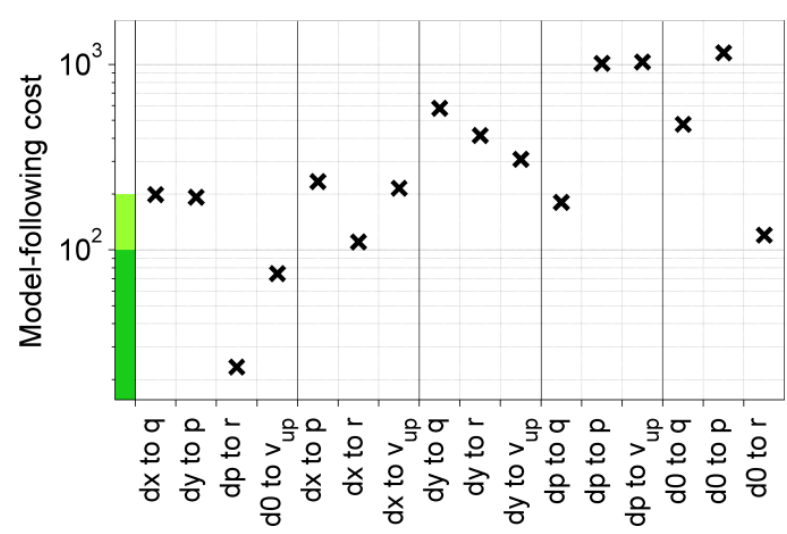

(a) Model-following costs based on sweep excitation signals with $5 \%$ amplitude.

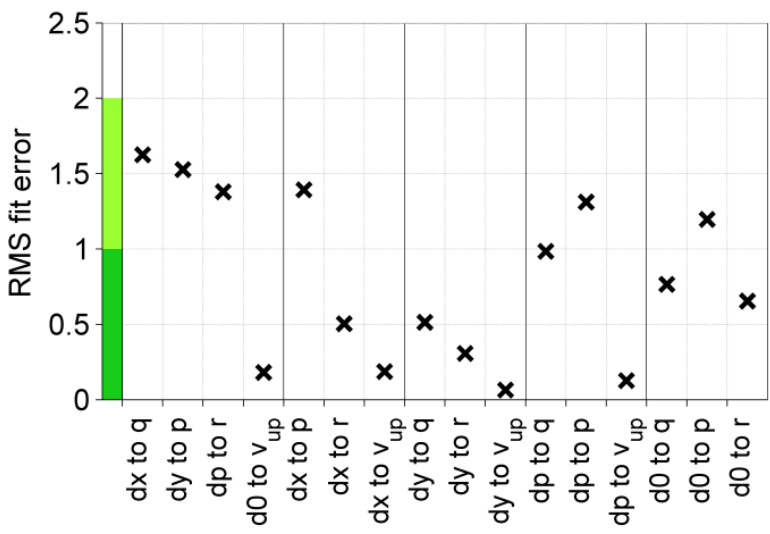

(b) RMS error, based on response data to doublets and 3-2-1-1 excitations with $2.5 \%, 5 \%$ and $10 \%$ amplitudes

Fig. 5 Cost in frequency-domain (a) and in time-domain (b) of each control/response axis. The error is calculated comparing the results in cases $B$ and $C$ of Fig. 4. The dark and light green sectors indicate an excellent and acceptable level of accuracy.

\section{Perceived fidelity evaluation}

The evaluation of the model-following precision has also been investigated subjectively by assessing the fidelity level of the in-flight simulation dynamics (case $\mathrm{C}$ of Fig. 4) against the command model dynamics (case B of Fig. 
4). To do so, pilots have been asked to fly evaluation maneuvers in AVES with these two configurations and to compare the respective dynamics. A total of eight maneuvers have been defined and flown by four pilots. These maneuvers were not chosen from the Mission Task Elements (MTE) defined in ADS-33 because most of these MTEs require either a full envelope model or at least a hover model and the model used in the command model is only valid around a forward velocity of 80 knots. The evaluation maneuvers are divided in two groups:

- Open-loop maneuvers: in these maneuvers, the pilot excites the helicopter using one control axis at a time and observes the four rate responses ( $\mathrm{p}, \mathrm{q}, \mathrm{r}, \mathrm{v}_{\text {up }}$ ). The excitations shall keep the helicopter around its trimmed velocity and shall have varied frequency content.

- Closed-loop Maneuvers: These maneuvers consist in basic maneuvers that are frequently flown by any helicopter pilot: acceleration /deceleration, 180deg turn, steady sideslip and climb/descent. The pilot can use each control axis to perform the maneuver as best as possible. The four rate responses and the controls applied shall be observed by the pilot during the maneuver.

After each maneuver has been flown in the two configurations, the pilot is requested to answer a questionnaire. In this questionnaire, the pilot assesses the level of flight dynamics dissimilarity between the reference and the test configurations using a 4-point Lickert-scale (negligible, minimal, moderate or considerable differences). The dynamics of the four rate responses are assessed individually. Additionally, a so-called "Simulator Fidelity Rating" (SFR) [24] is given to each of the closed-loop maneuvers. As a SFR can only be used to asses a maneuver as a whole, the Lickert-scale rating provides a complementary indication for the individual evaluation of each control/response axis. Those individual ratings can then be directly compared to the predictive fidelity metrics. An overview of the Lickert-scale ratings for all maneuvers can be seen in Fig. 6(a) and an overview of the SFR ratings for the closed-loop maneuvers can be seen in Fig. 6(b).

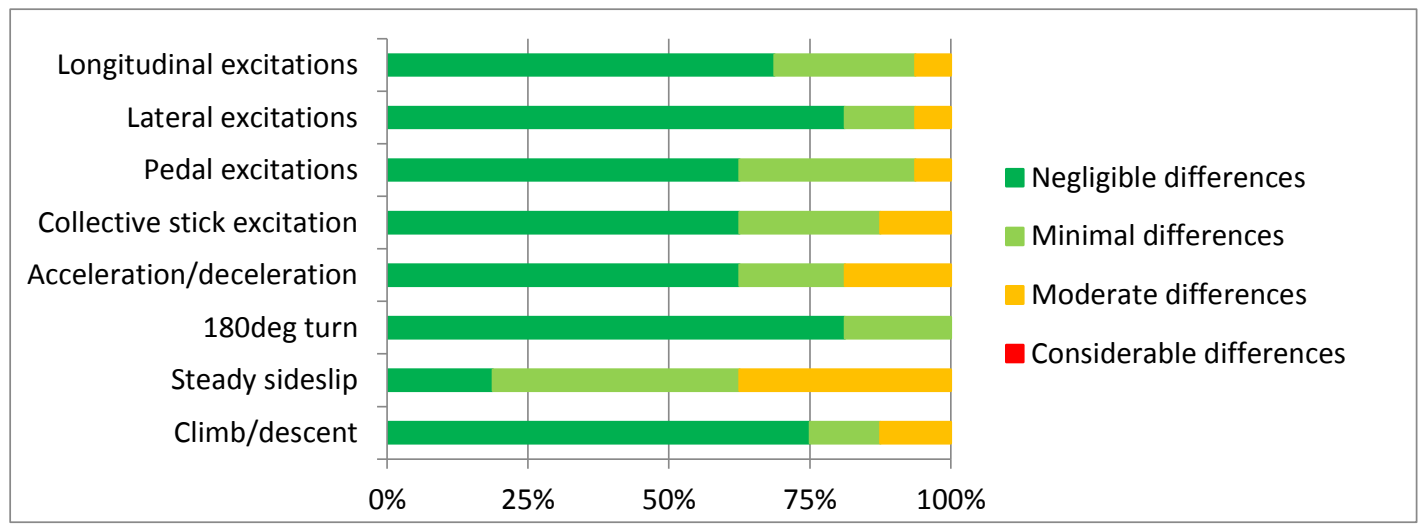

(a) Lickert-scale ratings: for each maneuver, the pilots rated the similarity between the two flight dynamics for each control/response axis. The individual ratings (four response axes for each pilot) are lumped in each maneuver for brevity.

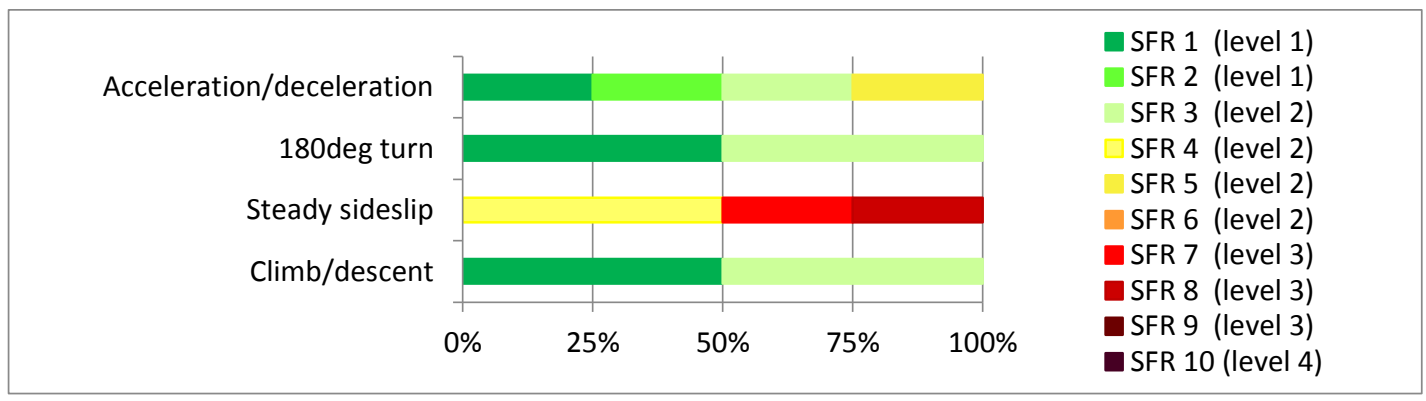

(b) Simulator fidelity ratings: One rating is attributed by each pilot to each closed-loop maneuver.

Fig. 6 Perceived fidelity evaluation of the in-flight simulation in AVES (case C of Fig. 4) when compared to the Bo-105 model (case B of Fig. 4).

Discussion of the results

The perceived fidelity evaluation can be summarized as follows. The flight dynamics differences between the command model and the in-flight simulation were rated as negligible or minimal in $87.5 \%$ of the 128 perceived 
fidelity evaluations performed (eight maneuvers, four pilots and four response axes). For the acceleration/deceleration, 180deg turn, and climb/descent maneuvers, the SFR ratings were very good: 11 out of the 12 ratings were between SFR 1 and SFR 3. The fidelity of the sideslip maneuvers was considered as not good. Two pilots gave a SFR of 7 and 8, corresponding to a fidelity level 3 (not fit for purpose). The reason of this poor fidelity is the following: the steady sideslip maneuver requires a constant bank angle and a constant lateral velocity. Since the stability derivative of the lateral velocity $Y_{\mathrm{v}}$ of the ACT/FHS is different from the one of the Bo-105, a same bank angle yields different lateral velocities. Therefore, a conflict occurs between the pilot, trying to keep a given bank angle, and the lateral feedback controller, trying to reach the commanded lateral velocity.

The predictive fidelity evaluation can be summarized as follows. The HQ analysis (Fig. 8) shows that the MBC successfully forced the ACT/FHS to follow the Bo-105 HQ characteristics. Indeed, all HQ levels calculated in this study were the same for the Bo-105 model and for the in-flight simulation; furthermore, the respective values were very close to each other. The only differences observed were an additional time delay of 50-100 $\mathrm{ms}$ and a small additional pitch coupling due to roll rate for the in-flight simulation. The time delay is the result of the inherent time delays in the control chain (filters, actuator delays, transport delays), and the small coupling difference is the result of the perfectible control precision for the off-axis responses. In the time- and frequency-domain each of the four onaxis responses gives a cost within the acceptable or excellent area (Fig. 5). For the off-axis responses the results differ between the time- and frequency-domain analyses: the yaw rate responses and heave velocity responses are mostly within the excellent area of the time-domain analysis, while they are partly far outside of the acceptable area in the frequency-domain analysis. The frequency-response function mismatches (Fig. 9) shows that the on-axis responses are mostly within the MUAD boundaries, but some of the off-axis responses are far outside of these boundaries. It is important to put the errors in frequency-domain into perspective with the absolute amplitude of the response. When the amplitude of a response function is very small, the frequency-response mismatch, which is a relative error, does not provide relevant information on the quality of the control precision. This is for example the case of the frequency mismatch of the following axes: dy to $r$, dy to $v_{u p}$, dp to $v_{u p}$. Those axes present an error in frequency-domain far outside of the boundaries, but their amplitude is very small and therefore, the impact on the absolute following error (Fig. 5(b)) is limited.

It is expected that the pitch and roll responses could be enhanced by tuning the feedback controller specifically for in-flight simulation. Indeed, one of the challenges of the feedback controller is to follow simultaneously the roll angle and the lateral velocity of the command model using the lateral cyclic; the same is true for the pitch angle and the longitudinal velocity with the longitudinal cyclic. As mentioned in section III, the feedback controller used in this study was tuned for a command model designed after ADS-33. With such a command model the difficulty to follow both the roll angle and lateral velocity is less of a problem since the stability derivatives of the lateral velocity can be chosen freely. In contrary, for in-flight simulation, these stability derivatives are constrained to have the value of the simulated helicopter. A typical solution is to follow the roll angle in the higher frequencies and the lateral velocity in the lower frequencies. This method is described under the name "frequency-dependent controller" in [25]. As the dynamics of the lateral velocity of the Bo-105 model is significantly different from the one of the ACT/FHS (the stability derivative of the lateral velocity $Y_{\mathrm{v}}$ in the Bo-105 model is about twice the value of the ACT/FHS), a reduction of the feedback gains of the lateral velocity might improve the model-following quality of the roll response.

\section{B. In-flight simulation fidelity evaluation}

This section describes the fidelity evaluation of the in-flight simulation in AVES compared to the real Bo- 105 . Due to the lack of measuring and recording systems in DLR's Bo-105, only the perceived fidelity evaluation was performed. The evaluation procedure was the same as the one used in the previous section. The only difference is that the reference case was a flight test with the Bo-105 (case A of Fig. 4) rather than a piloted simulation of the command model (case B of Fig. 4). Therefore, both the quality of the command model and the precision of the model-based controller are being evaluated. During the flight tests, the evaluation pilot was performing the maneuvers and giving the respective comments, while a safety pilot was monitoring the flight to prevent dangerous helicopter attitudes or traffic collisions. Two flight tests took place, involving two pilots; two more flight tests involving two other pilots are expected to take place in a near future. Each flight test took place on a day with little wind. The overview of the Lickert-scale and SFR ratings for all maneuvers can be seen in Fig. 7(a) and Fig. 7(b), respectively. 


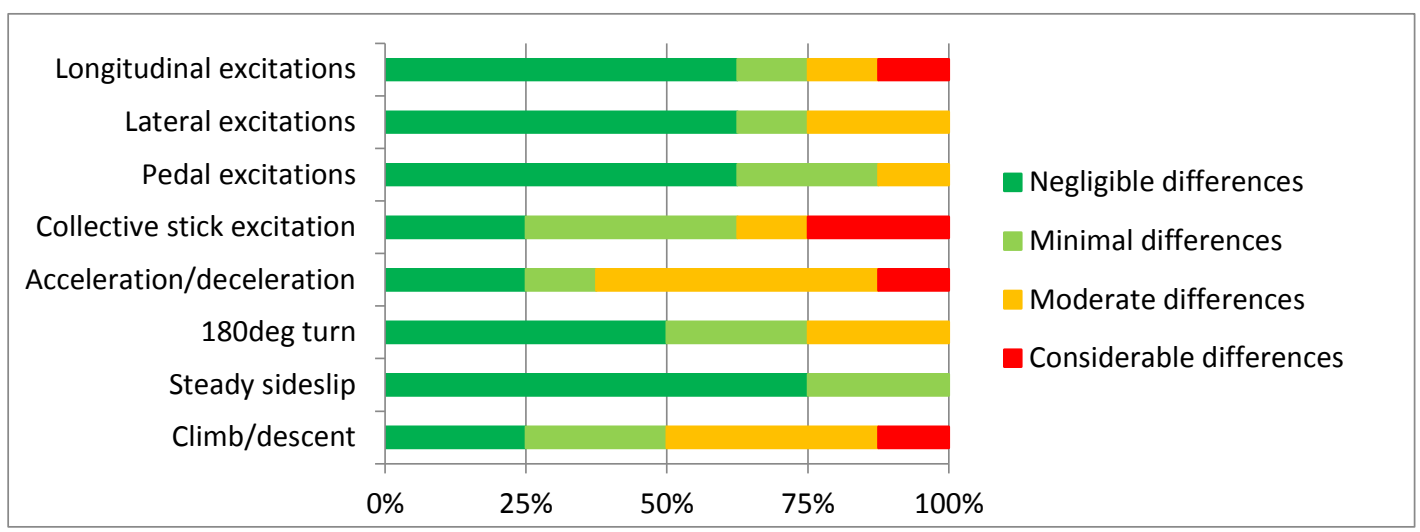

(a) Lickert-scale ratings: for each maneuver, the pilots rated the similarity between the two flight dynamics for each control/response axis. Here, the individual ratings (four response axes for each pilot) are lumped in each maneuver for brevity.

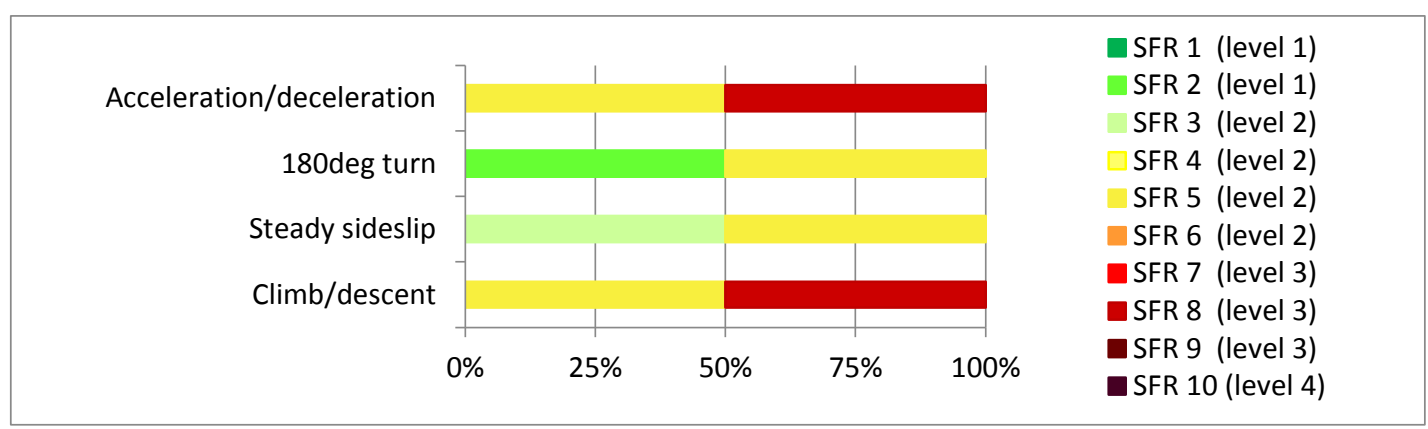

(b) Simulator fidelity ratings: one rating is attributed by each pilot to each closed-loop maneuver.

Fig. 7 Perceived fidelity evaluation of the in-flight simulation in AVES (case C of Fig. 4) when compared to the Bo-105 flight test (case A of Fig. 4).

\section{Discussion of the results}

As expected, the results are degraded when compared to the results of the previous section, since they additionally include the modeling errors of the Bo-105 helicopter. Nevertheless, both pilots mentioned that in almost all cases the reactions to control inputs, including the couplings, were in the good direction. The discrepancies were mostly related to the amplitude of the responses. The flight dynamics differences between the Bo-105 flight test and the in-flight simulation were considered as negligible or minimal in $70.3 \%$ of the 64 perceived fidelity ratings given (eight maneuvers, two pilots and four response axes), but $7.8 \%$ of them were considered as considerable. Most of the low fidelity ratings were linked to the off-axis responses to collective inputs. These deficiencies, clearly observed in the open-loop maneuvers, were also reflected in the closed-loop maneuvers: Out of the eight Simulator Fidelity Ratings (four closed-loop maneuvers with two pilots), five correspond to a Level 2 (Fidelity warrants improvements), two to a Level 3 (not fit for purpose) and one to a Level 1 (Fit for purpose). The two Level 3 ratings were attributed by one pilot to the acceleration/deceleration and to the climb/descent maneuvers. The reason mentioned by the pilot was that pitch, roll and yaw responses to the collective were not realistic. As large collective variations are applied during the acceleration/deceleration and the climb/descent maneuvers the fidelity of those maneuvers was degraded.

It is also important to note that the ratings given by the evaluation pilots showed important differences. It is not an easy task for a pilot to compare flight dynamics in two very different environments, such as a Bo-105 in flight and an EC-135 in a ground fixed-based simulator. The cockpit instruments are different, the sticks position, shape and dynamics are different, the motion is not present in the simulator, etc. 


\section{Conclusion}

In this paper, the Active Control Technology/ Flying Helicopter Simulator (ACT/FHS) [4] and its model-based controller (MBC) are briefly presented. A flight dynamics model of the Bo-105 at 80 knots tuned during piloted simulations is presented and its integration as a command model in the MBC is described. In-flight simulations were performed not in the ACT/FHS itself, but in the AVES simulator [16], using a fully nonlinear model of the ACT/FHS [17].

The precision of the MBC for each control/response axis is evaluated quantitatively through frequency-domain methods, time-domain methods and HQ analysis. A perceived fidelity evaluation process based on piloted simulations is presented and applied with four pilots in the AVES simulator. The evaluation demonstrates that the MBC can successfully transform the natural flight dynamics of the ACT/FHS into the flight dynamics of a 6-DoF linear Bo-105 model. Nevertheless, one specific maneuver (sideslip) obtains a low fidelity rating, probably due to the difficulty encountered by the feedback controller in following simultaneously the roll angle and the lateral velocity.

The fidelity of the in-flight simulation is evaluated comparing the flight dynamics of the Bo-105 during a flight test and of the in-flight simulation in the AVES simulator. A simulator fidelity level 2 is reached in most cases. The pilots mentioned that in almost all cases the reactions of the in-flight simulation to control inputs, including the couplings, were in the good direction. The discrepancies were mostly related to the amplitude of the responses. A significant deficiency of the Bo-105 model has been noticed for the off-axis responses to collective inputs, degrading the fidelity ratings for the maneuvers requiring important collective actions.

The demonstration of the In-flight simulations capability in the ACT/FHS is still pending.

Appendix

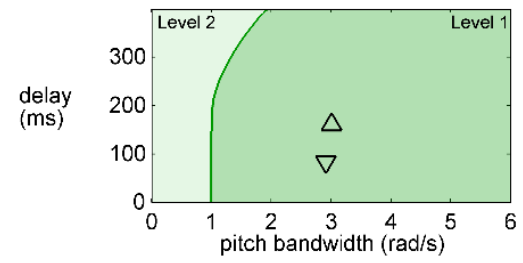

(a) Pitch Bandwidth

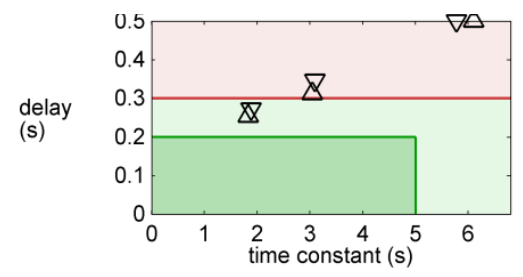

(d) Height Response

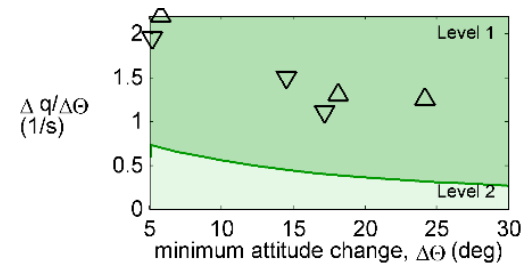

(g) Pitch Attitude Quickness

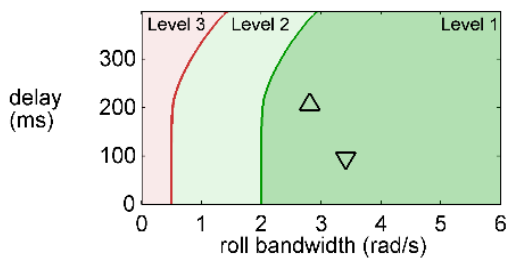

(b) Roll Bandwidth

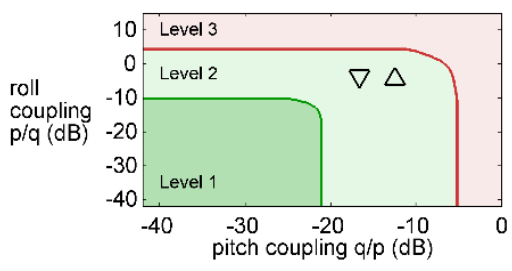

(e) Pitch-Roll-Coupling

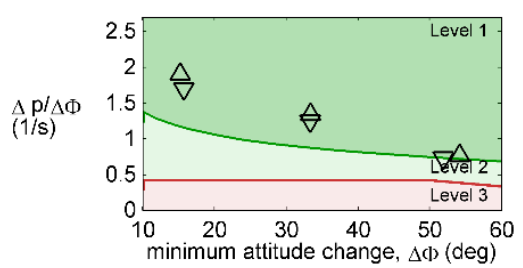

(h) Roll Attitude Quickness

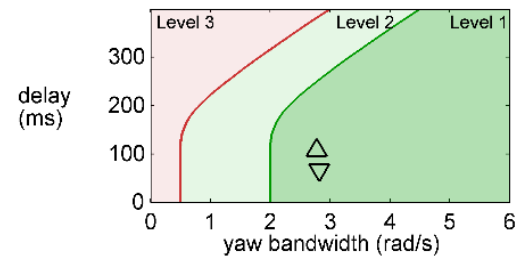

(c) Yaw Bandwidth

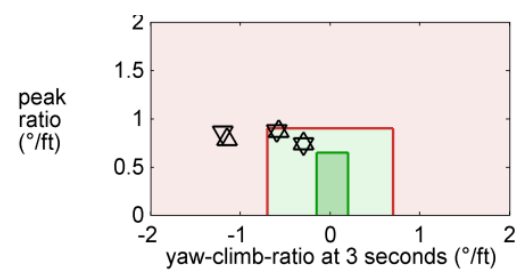

(f) Yaw-Collective-Coupling

Fig. 8 Handling Quality evaluation of the Bo-105 model (downwards-pointing triangles) and of the in-flight simulation in AVES (upwards-pointing triangles). 
From $d x$

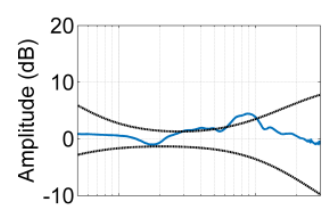

$\stackrel{0}{0}$

$\stackrel{2}{1}$
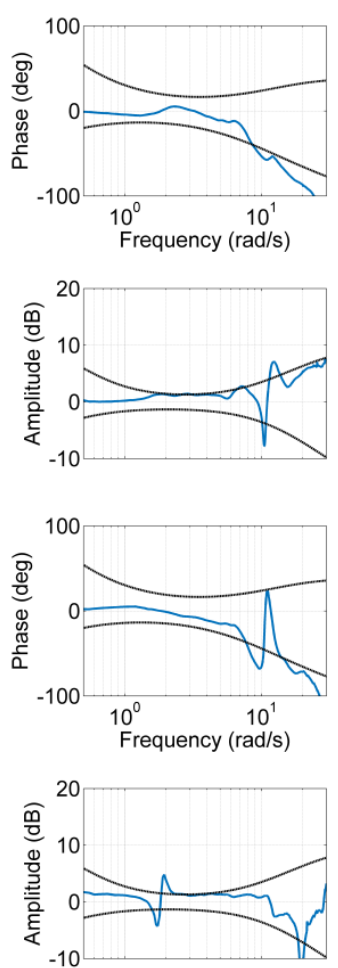

10
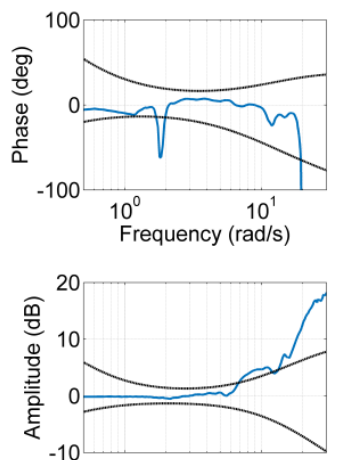

$\frac{5}{6}$

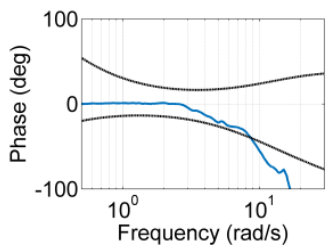

From dy
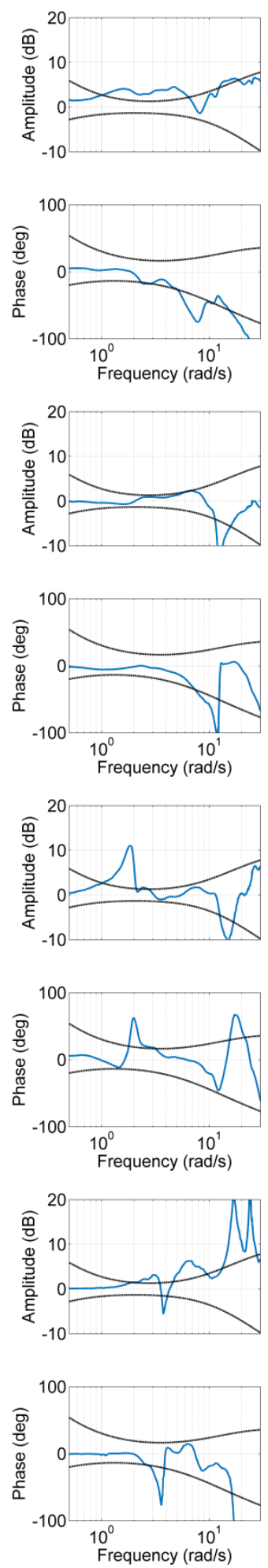

From dp
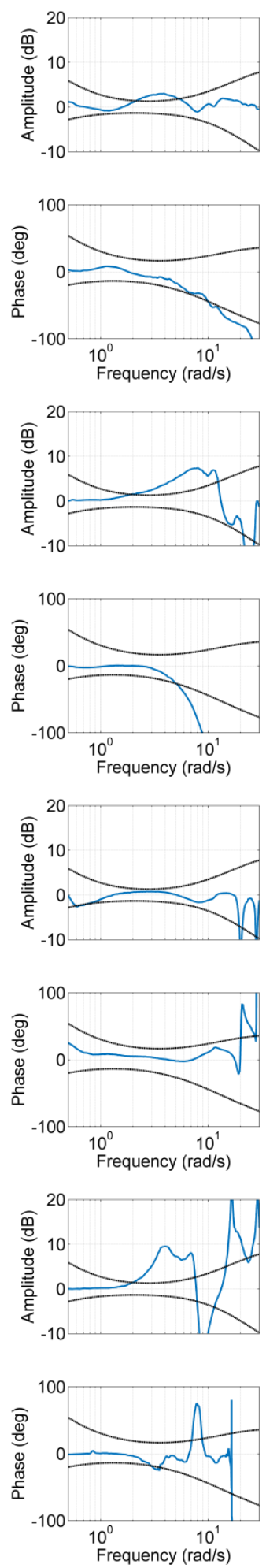

From d0
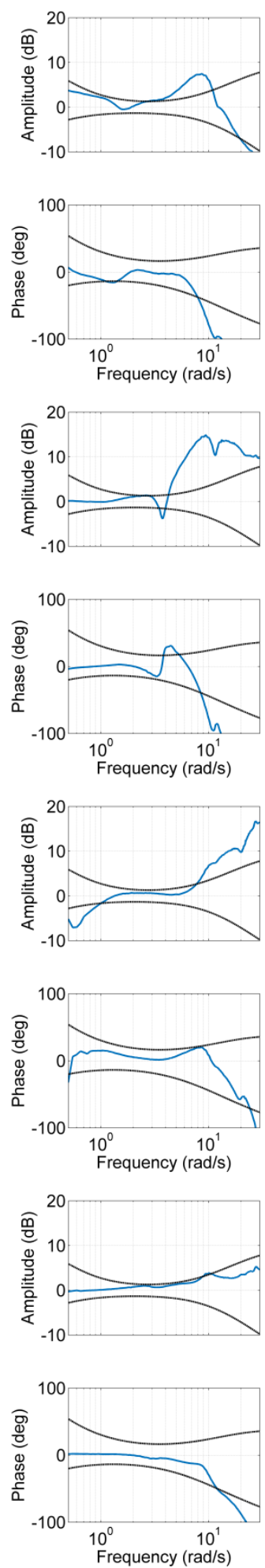

Fig. 9 FRF amplitude and phase mismatches (blue) between in-flight simulation in AVES and Bo-105 model, together with the MUAD envelope (black). 


\section{References}

[1] Hamel, P., G., In-Flight Simulators and Fly-by-Wire/Light Demonstrators, Springer, 2017.

[2] Moralez III, E., Hindson, W. S., Frost, C. R., Tucker, G. E., Arterburn, D. R., Kalinowski, K. F., and Dones, F., "Flight Research Qualification of the Army/NASA RASCAL Variable Stability Helicopter," American Helicopter Society 58th Annual Forum, Montréal, Canada, 2002.

[3] Gubbels, A. W., Carignan, S. J. R. P., and Ellis, D. K., "The NRC bell 412 advanced systems research aircraft - a new facility for airborne simulation," 1st AIAA, Aircraft, Technology Integration, and Operations Forum, 2001. doi: 10.2514/6.2001-5260

[4] Kaletka, J., Kurscheid, H., and Butter, U., "FHS, the New Research Helicopter: Ready for Service," Aerospace Science and Technology, vol. 9, Jul. 2005, pp. 456-467.

doi: 10.1016/j.ast.2005.02.003

[5] Bouwer, G., and Grünhagen, W. von, "LYNX Helicopter ln-Flight Simulation with ATTHeS", DLR IB 111-92/471992.

[6] Grünhagen, W. V., Bouwer, G., Pausder, H.-J., Henschel, F., and Kaletka, J., "A high bandwidth control system for a helicopter in-flight simulator,” International Journal of Control, vol. 59, 1994, pp. 239-261. doi: 10.1080/00207179408923077

[7] Fujizawa, B. T., "Control Law Design and Validation for a Helicopter In-flight Simulator," Master Thesis, California Polytechnic State University, 2010, URL: https://digitalcommons.calpoly.edu/theses/240/ [retrieved 15.09.2014].

[8] Lüken, T., and Korn, B., "PAVE: A prototype of a helicopter pilot assistant system," 33rd European Rotorcraft Forum, 2007, pp. 2567-2592.

[9] Greiser, S., Lantzsch, R., Wolfram, J., Wartmann, J., Müllhäuser, M., Lüken, T., Döhler, H.-U., and Peinecke, N., "Results of the pilot assistance system 'assisted Low-Level Flight and Landing on Unprepared Landing Sites' obtained with the ACT/FHS research rotorcraft," Aerospace Science and Technology, vol. 45, 2015, pp. 215-227. doi: 10.1016/j.ast.2015.05.017

[10] Gestwa, M., Wolfram, J., and al, "Projektplan ALL-In-Flight, Assisted Low Level Flight using In-Flight Simulation capability", DLR IB 111-2013/62, 2015.

[11] Wartmann, J., "Model validation and analysis using feedforward control flight test data," CEAS Aeronautical Journal, vol. 6, Sep. 2015, pp. 429-439.

[12] Rynaski, E. G., “Adaptative Multivariable Model Following,” Joint Automatic Control Conference., 1980, vol. 17, p.106.

[13] Greiser, S., and Lantzsch, R., "Equivalent modelling and suppression of air resonance for the ACT/FHS in flight," 39th European Rotorcraft Forum, 2013, pp. 376-387.

[14] Baskett, B. J., "Aeronautical Design Standard, Performance Specification, Handling Qualities Requirements for Military Rotorcraft", United States Army Aviation and Missile Command ADS33E-PRF, 2000, URL: http://www.dtic.mil/dtic/tr/fulltext/u2/a515904.pdf [retrieved 15.10.2014]

[15] Kaletka, J., Tischler, M. B., Grünhagen, W. von, and Fletcher, J. W., "Time and Frequency-domain identification and verification of BO-105 Dynamics Model," Journal of the American Helicopter Society, vol. 36, Oct. 1991, pp. $25-38$. doi: 10.4050/JAHS.36.25

[16] Duda, H., Gerlach, T., Advani, S. K., and Potter, M., "Design of the DLR AVES research flight simulator," AIAA Modeling and Simulation Technologies (MST) conference, Boston, MA, United States, 2013. doi: 10.2514/6.2013-4737

[17] Hamers, M., and Von Grunhagen, W., "Nonlinear helicopter model validation applied to realtime simulations," Annual Forum Proceedings - American Helicopter Society, 1997, pp. 958-972.

[18] Timson, E., "Flight Simulation Fidelity for Rotorcraft Design, Certification and Pilot Training", Ph.D. Dissertation, University of Liverpool, 2013, URL: https://livrepository.liverpool.ac.uk/15853/ [retrieved 20.11.2015].

[19] Wood, J. R., and Hodgkinson, J., "Definition of Acceptable Levels of Mismatch for Equivalent Systems of Augmented CTOL Aircraft", McDonnell Aircraft Company MDC-A6792, 1980.

[20] Cicolani, L. S., McCoy, A. H., Sahai, R., Tyson, P. H., Tischler, M. B., Rosen, A., and Tucker, G. E., "Flight Test Identification and Simulation of a UH-60A Helicopter and Slung Load," Journal of the American Helicopter Society, vol. 46, Apr. 2001, pp. 140-160.

doi: 10.4050/JAHS.46.140

[21] Strope, K., Borden, C., and Harding, J., "Verification \& Validation of a UH-60 FLIGHTLAB model in support of the UH-60M Limited User Test," American Helicopter Society 60th Annual Forum, Baltimore, MD, 2004.

[22] Mitchell, D. G., Hoh, R. H., He, C., and Strope, K., "Determination of Maximum Unnoticeable Added Dynamics," AIAA Atmospheric Flight Mechanics Conference and Exhibit, Keystone, Colorado, 2006. doi: 10.2514/6.2006-6492

[23] Tischler, M. B., and Remple, R. K., Aircraft and Rotorcraft System Identification: Engineering Methods with Flight-Test Examples, $2^{\text {nd }}$ ed., Reston, Virginia, USA: American Institute of Aeronautics and Astronautics, Inc., 2012. doi: 10.1109/MCS.2015.2512078

[24] Perfect, P., Timson, E., White, M. D., Padfield, G. D., Erdos, R., and Gubbels, A. W., "A Rating Scale for Subjective Assessment of Simulator Fidelity," 37th European Rotorcraft Forum, Gallarate, Italy, 2011, pp. 43-47.

[25] Fujizawa, B. T., Ivler, C. M., Tischler, M. B., Moralez, E., and Braddom, S. R., "In-Flight Simulation Control Law Design and Validation for RASCAL," AHS International 66th Annual Forum, Phoenix, AZ, 2010. 